\title{
Disease Inference and Community-based Health Services
}

\author{
Asif Akram ${ }^{1}$, Rekha Jadhav ${ }^{2}$ \\ ${ }^{1}$ Master of Computer Engg, Savitribai Phule Pune University, G. H. Raisoni Collage of Engg and Techonology, Wagholi, Pune \\ ${ }^{2}$ HOD, IT Dept, Savitribai Phule Pune University, G. H .Raisoni Collage of Engg and Techonology, Wagholi, Pune
}

\begin{abstract}
Automatic disease inference is of significance to overcome any issues between what online health seekers with strange side effects need and what occupied human doctor with one-sided aptitude can offer. In any case, precisely and productively surmising infections is non-trivial, particularly for group based health administrations because of the vocabulary crevice, fragmented information, related medicinal ideas, and constrained brilliant preparing tests. In this paper, we first report a client study on the information needs of health seekers as far as inquiries and afterward select those that request conceivable illnesses of their showed side effects for further explanatory. We next propose a novel profound learning plan to deduce the conceivable maladies given the inquiries of health seekers. The proposed plan involves two key parts. The principal all around mines the separate restorative marks from crude components. The second esteems the crude elements and their marks as information nodes in one layer and hidden nodes in the ensuing layer, individually. Then, it takes in the between relations between these two layers by means of preparing with pseudo labeled data. Taking after that, the shrouded hubs serve as crude elements for the more theoretical mark mining. With incremental and option rehashing of these two parts, our plan fabricates an inadequately joined deep architecture with three hidden layers. By and large, it well fits particular errands with calibrating. Broad trials on a certifiable dataset named by online specialists demonstrate the noteworthy execution additions of our plan.
\end{abstract}

Keywords: Community-based Health Services, Question Answering, Disease Inference, Deep Learning

\section{Introduction}

The turning gray of society, heightening expenses of medicinal services furthermore, thriving PC advances are as one driving more shoppers to invest longer energy online to investigate health data. One study in [1] demonstrates that $59 \%$ of U.S. grown-ups have investigated the web as a symptomatic instrument in 2012. Another review in [2] reports that the normal U.S. shopper spends near 52 hours every year online to discover health learning, while just visits the specialists three times every year in 2013. The current programmed question answering procedures are relevant here [3], [4]. The disease inference execution can be gotten by general shallow learning strategies. Shallow learning systems allude to the most advanced learning calculations, for example, decision trees, and bolster vector machine (SVM), where the yield of a learning plan is specifically trailed by a classified as though the framework has one and only layer.

In this paper we manufacture an infection deduction plot that has the capacity consequently surmise the conceivable ailments of the given inquiries in group based wellbeing administrations. We first break down and arrange the data needs of wellbeing seekers. As a repercussion, we separate inquiries of this kind that require illness derivation from different sorts. It merits underscoring that large-scale data frequently prompts blast of highlight space in the lights of ngram representation [5] [6] particularly for the group created conflicting data. To keep away from this issue, we use the medicinal phrasings to speak to our information. Our plan fabricates a novel profound learning model, containing two parts. The primary all around mines the inactive restorative marks. They are reduced examples of between subordinate restorative wordings or crude elements, which can surmise the deficient data. The crude components and marks individually serve as info hubs in one layer and shrouded hubs in the resulting layer. The second takes in the interrelations between these two layers through prepreparing. Taking after that, the concealed nodes are seen as crude highlights for more dynamic mark mining. With incremental and option rehashing of these two parts, our plan assembles a meagerly associated profound learning structural planning with three concealed layers. This model is generalizable and versatile. Adjusting with a little arrangement of marked sickness tests fits our model to particular malady surmising. Not the same as traditional profound learning calculations, the quantity of concealed nodes in every layer of our model is consequently decided and the associations between two nearby layers are inadequate, which make it quicker. Broad examinations on certifiable dataset named by online specialists were directed to approve our plan.

\section{Literature Survey}

1) A Classification-based Approach to Question Routing in Community Question Answering

Author: Tom Chao Zhou, Michael R. Lyu, Irwin King

Community based Question and Answering (CQA) administrations have conveyed clients to another time of information spread by permitting clients to make inquiries and to answer other clients' questions. In any case, because of the quick expanding of posted questions and the absence of a compelling approach to discover intriguing questions, there is a genuine crevice between posted questions what's more, potential answerers. This hole may debase a CQA administration's execution and additionally lessen clients' reliability to the framework. To conquer any hindrance, we show another way to deal with Inquiry Routing, which goes for directing inquiries to members why should likely give answers. We consider the issue of inquiry steering as an arrangement assignment, and add to an assortment of nearby and worldwide elements which catch distinctive parts of inquiries, clients, and their relations. Author likewise perform a systematical 


\section{International Journal of Science and Research (IJSR) \\ ISSN (Online): 2319-7064}

Index Copernicus Value (2013): 6.14 | Impact Factor (2014): 5.611

correlation on how distinctive sorts of components add to the last results what's more; demonstrate that question-client relationship components play a key part in enhancing the general execution.

\section{2) Multimedia Answering: Enriching Text QA with Me- dia Information}

Author: Liqiang Nie, Meng Wang, Zheng-Jun Zha, Guangda Li and Tat-Seng Chua

Existing community question-answering discussions more often than not give just printed answers. On the other hand, for some inquiries, immaculate writings can't give instinctive data, while picture then again video substance are more suitable. In this paper, author present a plan that can advance content answers with picture and video data. Our plan examines a rich set of methods including inquiry/answer grouping, question era, picture and video pursuit reranking, and so on.Given an inquiry and the group contributed answer, authors methodology can figure out which sort of media data should be included, and afterward naturally gathers information from Internet to improve the printed answer. Distinctive from a few endeavors that endeavor to specifically answer questions with picture and video information, our methodology is based in view of the group contributed literary answers and hence it is more plausible and ready to manage more mind boggling inquiries. Authors have led exact study on more than 3,000 QA sets and the outcomes exhibit the adequacy of our approach.

\section{3) Beyond Text QA: Multimedia Answer Generation by Harvesting Web Information}

Author: Liqiang Nie, Meng Wang

Community question answering (cQA) administrations have picked up prevalence over the previous years. It not just permits group individuals to post and answer addresses additionally empowers general clients to look for data froma exhaustive arrangement of all around replied questions. Be that as it may, existing cQA discussions as a rule give just printed answers, which are not sufficiently instructive for some questions. In this paper, author propose a plan that can enhance printed answers in cQA with fitting media information. author plan comprises of three segments: answer medium choice, question era for media inquiry, and mixed media information determination furthermore, presentation. This methodology naturally decides which kind of media data ought to be included for a printed answer. It then consequently gathers information from the web to improve the answer. By preparing a substantial arrangement of QA matches and adding them to a pool, our methodology can empower a novel interactive media inquiry replying (MMQA) approach as clients can discover sight and sound answers by coordinating their inquiries with those in the pool. Not the same as a parcel ofMMQAresearch endeavors that endeavor to specifically answer questions with picture and video information, our methodology is based in view of group contributed printed answers and in this manner it can bargain with more mind boggling questions. We have led broad investigations on a multi-source QA dataset. The outcomes exhibit the viability of our methodology.

\section{4) Learning to Recommend Descriptive Tags for Ques- tions in Social Forums}

Author: LIQIANG NIE, YI-LIANG ZHAO
Around $40 \%$ of the questions in the developing socialoriented question answering discussions have at generally one physically marked label, which is brought on by incomprehensive question understanding or casual labeling practices. The deficiency of inquiry labels seriously impedes all the tag-based controls, for example, nourishes for point supporters, ontological learning association, and other fundamental insights. This article presents a novel plan that can exhaustively learn unmistakable labels for every question. Broad assessments on an agent certifiable dataset exhibit that our plan yields noteworthy additions for question annotation, and all the more imperatively, the entire procedure of our methodology is unsupervised and can be stretched out to handle large-scale data.

\section{5) Extracting KeySubstringGroup Features for Text Classification}

Author: Dell Zhang, Wee Sun Lee

In numerous text classification applications, it is engaging take each archive as a series of characters as opposed to a sack of words. Past examination thinks about around there for the most part concentrated on distinctive variations of generative Markov chain models. Albeit discriminative machine learning strategies like Support Vector Machine (SVM) have been very fruitful in content grouping with word highlights, it is not one or the other viable nor productive to apply them clearly taking all substrings in the corpus as elements. In this paper, author propose to parcel all substrings into measurable comparability gatherings, and afterward pick those gatherings which are essential (in the measurable sense) as components for content grouping. Specifically, author is proposing an addition tree based calculation that can remove such elements in direct time. Authors are examinations on English, Chinese and Greek datasets demonstrate that SVM with key-substring-gathering components can accomplish exceptional execution for different content grouping assignments.

\section{6) The Bag-of-Repeats Representation of Documents} Author: Matthias Galle

n-gram representations of reports may enhance over a basic bag-of-word representation by unwinding the independence suspicion of word and presenting setting. However, this includes some significant downfalls of including components which are nonengaging, and expanding the measurement of the vector space model exponentially. Author is show new representations that maintain a strategic distance from both pitfalls. They depend on sound hypothetical thoughts of stringology, what's more, can be figured in ideal asymptotic time with algorithms utilizing information structures from the su_x family. While maximal rehashes have been utilized as a part of the past for comparable undertakings, author indicate how another identicalness class of rehashes - biggest maximal rehashes - get comparative or better results, with just a small amount of the elements. This example is excellence as an insignificant generative premise of every rehashed substring. We likewise report their use for subject demonstrating, indicating simpler to decipher models.

\section{Existing System}

Disease inference is a thinking outcome in view of the given inquiries; this undertaking is non-trifling because of taking 


\section{International Journal of Science and Research (IJSR) \\ ISSN (Online): 2319-7064}

Index Copernicus Value (2013): 6.14 | Impact Factor (2014): 5.611

after reasons. To begin with, vocabulary hole between various health seekers makes the data more conflicting, when contrasted with different configurations of health data. Second, health seekers portray their issues in short inquiries, containing 14.5 terms for each inquiry overall. The fragmentation upsets the compelling closeness estimation taking into account shared settings. Third, restorative traits, for example, age, sex and manifestations, are exceedingly associated and don't bizarrely show up as minimal examples to flag the health issues. Moreover, it is costly to develop the ground truth for different diseases.

\section{Disadvantages}

Existing system limit the disease inference performance that can be obtained by general shallow learning methods.

\section{Proposed System}

The proposed plan involves two key parts. The main all inclusive mines the separate medicinal marks from crude elements. The second regards the crude elements and their marks as info nodes in one layer and shrouded nodes in the consequent layer, individually. In the interim, it takes in the between relations between these two layers through prepreparing with pseudolabeled data. Taking after that, the concealed hubs serve as crude elements for the more theoretical mark mining. With incremental and option rehashing of these two segments, our plan fabricates a meagerly joined deep architecture with three hidden layers. Generally speaking, it well fits particular undertakings with tweaking. Broad investigations on a certifiable dataset named by online specialists demonstrate the critical execution additions of our plan.

\section{Advantages}

It is capable of handling various kinds of diseases effectively.

\section{Modules}

\section{- Signature Mining}

The medical signatures are more graphic than raw feature and will fundamentally diminish the measurement of highlight space. In any case, it is hard to concentrate such signature from individual information cases, as their structures are typically implicitly distributed over an expansive scale dataset.

\section{- Disease Inference}

Deep learning plan is to deduce the possible diseases given the question of health seekers. Contrasted with shallow adapting, deep learning has a few focal points. To start with, it can learn delegate and versatile elements from other infection sorts [7]. Take the lung cancer induction learning as a case. At the point when building its classifier, the preparation information can be liver tumor or other infection tests as opposed to entirely compelled to lung tumor. This locations the restricted ground truth and need of malady mindful element extraction [8]. Second, acquired from its profound architectures, it over and over takes in the more conceptual minimized examples layer by layer.

\section{- Sparsely Connected Deep Learning}

Nodes in the higher layer are the signatures of and join with the hubs in its nearby lower layer, as opposed to completely associated. These relations are unequivocally demonstrated by the liking network, which remains for the connection quality between node $i$ in layer 1 and node $j$ in its consequent higher layer.

\section{- Optimization}

This parameter decides how quick or moderate we will move towards the ideal qualities. In the event that it is too little we will need an excess of emphasess to join to the ideal values. In light of the angle plummet, we iteratively overhaul the parameters with introductions until the predefined end foundations are met.

\section{- Pre-training and Fine-tuning}

The quality of unsupervised pre-training to deep learning has been demonstrated [9]. Our model holds three scantily snared concealed layers and they are pre-trained consecutively. We develop a chart over our entire dataset in light of the fundamental associations among the crude components. We next mine the marks from this chart by means of thick subgraph recognition. These marks are seen as concealed hubs and put in the first shrouded layer.

\section{Conclusion}

This paper initially performed client study to break down the health seeker needs. This gives the bits of knowledge of group based health services. It then exhibited a meagerly associated profound learning plan that is ready to gather the conceivable maladies given the inquiries of health seekers. This plan is built by means of alternative signature mining and pre-preparing in an incremental way. It licenses unsupervised feature learning from other extensive variety of infection sorts. In this way, it is generalizable and versatile as thought about to past infection surmising utilizing shallow learning approaches, which are normally prepared on doctor's facility created patient records with organized fields Traditional profound learning architectures are thickly joined and the hub number in each covered up layers are drearily balanced. In contract, our model is inadequately associated with enhanced learning productivity; furthermore, the quantity of hidden nodes is naturally decided.

\section{References}

[1] Disease Inference from Health-Related Questions via Sparse Deep Learning Liqiang Nie, Meng Wang, Luming Zhang, Shuicheng Yan, Member, IEEE, Bo Zhang, Senior Member, IEEE, Tat-Seng Chua, Senior Member, IEEE

[2] "Online health research eclipsing patient-doctor conversations," Makovsky Health and Kelton, Survey, 2013.

[3] L. Nie, M. Wang, Z. Zha, G. Li, and T.-S. Chua, "Multimedia answering: Enriching text qa with media information," in Proceedings of the International ACM SIGIR Conference, 2011.

[4] L. Nie, M. Wang, Y. Gao, Z.-J. Zha, and T.-S. Chua, "Beyond text qa: Multimedia answer generation by harvesting web information," Multimedia, IEEE Transactions on, 2013. 


\section{International Journal of Science and Research (IJSR) \\ ISSN (Online): 2319-7064}

Index Copernicus Value (2013): 6.14 | Impact Factor (2014): 5.611

[5] D. Zhang and W. S. Lee, "Extracting key-substring-group features for text classification," in The ACM SIGKDD Conference on Knowledge Discovery and Data Mining, 2006.

[6] M. Gall'e, "The bag-of-repeats representation of documents," in Proceedings of the International ACM SIGIR Conference, 2013.

[7] Y. Bengio, A. Courville, and P. Vincent, "Representation learning: A review and new perspectives," IEEE Transactions on Pattern Analysis and Machine Intelligence, 2013.

[8] R. Fakoor, F. Ladhak, A. Nazi, and M. Huber, "Using deep learning to enhance cancer diagnosis and classification," in Proceedings of the International Conference on Machine Learning, 2013

[9] D. Erhan, Y. Bengio, A. Courville, P.-A. Manzagol, P. Vincent, and S. Bengio, "Why does unsupervised pretraining help deep learning?" Journal of Machine Learning Research, 2010 